\title{
On the Distributions of Logarithmic Derivative of Differentiable Measures on $\boldsymbol{R}$
}

By

Hiroaki SHImomura*

In [1], Hora discussed distributions of logarithmic derivative of differentiable probability measures on $\boldsymbol{R}$ and obtained the following theorem with Yamasaki.

Theorem. Let $P$ be an arbitrary probability distribution with mean 0 which is not Dirac measure $\delta_{0}$ at 0 . Then there exists some differentiable probability measure $d \mu(x)=f(x) d x$ such that $P(E)=\mu\left(x \mid f^{\prime}(x) / f(x) \in E\right)$ for all $E \in \mathfrak{B}(\boldsymbol{R})$, where $d x$ is the Lebesgue measure on $\boldsymbol{R}$ and $\mathfrak{B}(\boldsymbol{R})$ is the usual Borel field on $\boldsymbol{R}$.

In this note, we will give a simple proof of this theorem and add a few comments. First we shall supplement some definitions and a few facts. (See, [1] and [2]).

(a) A probability measure $\mu$ is said to be differentiable, if $\mu(E-t)$ is a differentiable function of $t$ for each $E \in \mathfrak{B}(\boldsymbol{R})$.

(b) For the differentiability of $\mu$, it is necessary and sufficient that (1) $\mu$ is absolutely continuous with $d x$ and (2) its density $f(x)$ is differentiable almost everywhere on $\boldsymbol{R}$ and $f^{\prime}(x) \in L_{d x}^{1}(\boldsymbol{R})$.

(c) If $\delta_{0}$ would coincide with the distribution $\mu_{f}$ of logarithmic derivative $f^{\prime} / f$ of $\mu(d \mu(x)=f(x) d x)$, then it follows that $f^{\prime}=0$ almost everywhere and that $f \equiv 0$. Thus we must exclude the case $P=\delta_{0}$ for this problem.

(d) The distribution $\mu_{f}$ has mean 0 . Therefore we must consider only probability distributions $P$ with mean 0 .

Before beginning the proof of the Theorem, we wish to state some idea which is somewhat formal. For a given $P$ define a function $\omega(t)$ on $(0,1)$ such that $\omega(t)=\sup \{x \in \boldsymbol{R} \mid P((-\infty, x)) \leqq t\}$. Then $\omega$ is increasing and by the properties of supremum,

$$
P((-\infty, \omega(t))) \leqq t \quad \text { for all } t \in(0,1) \text {, and }
$$

Communicated by S. Matsuura, June 2, 1988.

* Department of Mathematics, Fukui University, Fukui 910, Japan. 


$$
P((-\infty, x))>t, \quad \text { if } \quad x>\omega(t) .
$$

Now let $\lambda$ be the Lebesgue measure on $[0,1]$ and define a measure $\omega \lambda$ on $\mathfrak{B}(\boldsymbol{R})$ such that $\omega \lambda(E)=\lambda(t \mid \omega(t) \in E)$ for all $E \in \mathfrak{B}(\boldsymbol{R})$. It follows from (2) that $\omega \lambda((-\infty, x))=P((-\infty, x))$ for all $x \in \boldsymbol{R}$. So we have

$$
\omega \lambda=P \text {. }
$$

Consequently,

$$
\begin{gathered}
\int_{0}^{1}|\omega(t)| d \lambda(t)=\int_{-\infty}^{\infty}|x| d P(x)<\infty, \quad \text { and } \\
\int_{0}^{1} \omega(t) d \lambda(t)=\int_{-\infty}^{\infty} x d P(x)=0 .
\end{gathered}
$$

Thus the problem is to find $f$ which satisfies $\int_{-\infty}^{\infty} \chi_{E}\left(f^{\prime}(x) / f(x)\right) f(x) d x=$ $\int_{0}^{1} \chi_{E}(\omega(t)) d t$, where $\chi_{E}$ is the indicator function of any Borel set $E$.

In order to find such $f$, we rewrite the right hand side using integration by substitution with a suitable monotone differentiable function $\gamma$ on $(0,1)$. After some calculations (which is omitted here) we reach to a contradiction in the case that $\gamma$ is strictly increasing. On the other hand if $\gamma$ is strictly decreasing, then putting $\lim _{t \rightarrow 1} \gamma(t)=\alpha, \quad \lim _{t \rightarrow 0} \gamma(t)=\beta$, we have $\int_{0}^{1} \chi_{E}(\omega(t)) d t=$ $-\int_{\alpha}^{\beta} \chi_{E}\left(\omega\left(\gamma^{-1}(x)\right)\left(\gamma^{-1}(x)\right)^{\prime} d x\right.$. So if we take

$$
\begin{aligned}
& f^{\prime}(x) / f(x)=\omega\left(\gamma^{-1}(x)\right), \quad \text { and } \\
& f(x)=-\left(\gamma^{-1}(x)\right)^{\prime}=\frac{-1}{\gamma^{\prime}\left(\gamma^{-1}(x)\right)},
\end{aligned}
$$

then the both sides in the above equality have the same form except the lower limit and upper limit of integration. From (6) and (7) it follows that $\omega\left(\gamma^{-1}(x)\right)$ $=-\gamma^{\prime}\left(\gamma^{-1}(x)\right) f^{\prime}(x)$ and therefore $\omega(t)=-(f \circ \gamma)^{\prime}(t)$. Thus for a function defined by $h(t)=\int_{0}^{t} \omega(\tau) d \tau$, we have $f(\gamma(t))=-h(t)+$ const and this constant must be 0 , because $f(x)$ must satisfy $\lim _{x \rightarrow \pm \infty} f(x)=0$. Further it follows from $(7) \gamma^{\prime}(t)=h(t)^{-1}$ and $\gamma(t)=\int_{1 / 2}^{t} \frac{\lceil d \tau}{h(\tau)}+$ const. From now on we shall show that this procedure actually gives the desired function $f$.

\section{(Proof of Theorem)}

It is clear that $h(t) \equiv \int_{0}^{t} \omega(\tau) d \tau$ is absolutely continuous, and that $h(0)=h(1)$ $=0$. $h(t)$ is negative on $(0,1)$. In fact suppose that $h(t)$ would be 0 for some $t_{0} \in(0,1)$. Then $0=h(1)-h\left(t_{0}\right)=\int_{t_{0}}^{1} \omega(\tau) d \tau \geqq \omega\left(t_{0}\right)\left(1-t_{0}\right)$, which shows $\omega\left(t_{0}\right) \leqq 0$. Similarly $0=h\left(t_{0}\right)-h(0)$ shows $\omega\left(t_{0}\right) \geqq 0$, hence $\omega\left(t_{0}\right)=0$. Again from $0=h(1)-$ 
$h\left(t_{0}\right)=h\left(t_{0}\right)-h(0)$, we have $\omega(\tau) \equiv 0$ on $(0,1)$, which contradicts to $P \neq \delta_{0}$. As $\omega(\tau)$ is negative for sufficiently small $\tau, h(t)$ is negative on $(0,1)$. Now we can define a function $\gamma$ on $(0,1)$ such that $\gamma(t)=\int_{1 / 2}^{t} \frac{d \lambda(\tau)}{h(\tau)}$. Then $\gamma$ is strictly decreasing continuously differentiable function on $(0,1)$. Put $\lim _{t \rightarrow 1} \gamma(t)=\alpha(\geqq-\infty)$ and $\lim _{t \rightarrow 0} \gamma(t)=\beta(\leqq \infty)$. Lastly we define a function $f(x)$ on $\boldsymbol{R}$ such that $f(x)$ $=-h\left(\gamma^{-1}(x)\right)$, if $x \in(\alpha, \beta)$ and $f(x)=0$, otherwise. Since $f$ is absolutely continuous on any closed interval of $(\alpha, \beta)$ and $\lim _{x \rightarrow \alpha} f(x)=\lim _{x \rightarrow \beta} f(x)=0$, so it is continuous, differentiable almost everywhere and

$$
f^{\prime}(x)=-\omega\left(\gamma^{-1}(x)\right) h\left(\gamma^{-1}(x)\right)=\omega\left(\gamma^{-1}(x)\right) f(x) \quad \text { on }(\alpha, \beta) .
$$

Then

$$
\begin{aligned}
& \int_{-\infty}^{\infty} f(x) d x=-\int_{a}^{\beta} h\left(\gamma^{-1}(x)\right) d x=\int_{0}^{1} h(t) \gamma^{\prime}(t) d \lambda(t)=1, \quad \text { and } \\
& \left.\int_{-\infty}^{\infty}\left|f^{\prime}(x)\right| d x=\int_{a}^{\beta} \mid \omega^{\prime} \gamma^{-1}(x)\right)\left|f(x) d x=\int_{0}^{1}\right| \omega(t) \mid d \lambda(t)<\infty .
\end{aligned}
$$

Consequently $f(x)$ is an absolutely continuous function on $\boldsymbol{R}$ and a measure defined by $d \mu(x)=f(x) d x$ is differentiable. Now we have $\left.\mu(x) f^{\prime}(x) / f(x) \in E\right)=$ $-\int_{\alpha}^{\beta} \chi_{E}\left(\omega\left(\gamma^{-1}(x)\right) h\left(\gamma^{-1}(x)\right) d x=\int_{0}^{1} \chi_{E}(\omega(t)) d \lambda(t)=P(E)\right.$ for all Borel sets $E$.

Q.E.D.

Remark 1. $f_{k}(x) \equiv f(x+k)$ ( $k$ : an arbitrary constant) also satisfies $\mu_{f_{k}}=P$, because the translation of $f$ does not change the distribution of logarithmic derivative.

Remark 2. If $P$ is a symmetric distribution i.e., $P(E)=P(-E)$ for all $E \in \mathfrak{B}(\boldsymbol{R})$, then $f$ is an even function and $f(0)>0$.

Proof. Take any $t \in(0,1 / 2)$. Then $P((-\infty, \omega(t+1 / 2)+\varepsilon))>t+1 / 2$ and $P((-\varepsilon-\omega(1 / 2-t), \infty))=P((-\infty, \omega(1 / 2-t)+\varepsilon))>1 / 2-t$. It follows that $\omega(t+1 / 2)$ $+\varepsilon>-\varepsilon-\omega(1 / 2-t)$ for all $\varepsilon>0$ and hence $\omega(t+1 / 2)+\omega(1 / 2-t) \geqq 0$. Since $0=\int_{0}^{1} \omega(t) d \lambda(t)=\int_{0}^{1 / 2}\{\omega(t+1 / 2)+\omega(1 / 2-t)\} d \lambda(t)$, so $\omega(t+1 / 2)+\omega(1 / 2-t)=0$ for almost all $t \in(0,1 / 2)$. Consequently it follows from (5) $h(t+1 / 2)=h(1 / 2-t)$ and from this $\gamma(t+1 / 2)=-\gamma(1 / 2-t)$ for all $t \in(0,1 / 2)$. Thus we have $f(0)=-h\left(\gamma^{-1}(0)\right)$ $=-h(1 / 2)>0$, and $f(\gamma(t+1 / 2))=-h(t+1 / 2)=-h(1 / 2-t)=f(\gamma(1 / 2-t))=f(-\gamma(t+$ $1 / 2))$.

Q.E. D.

Conversely, it is evident that if $f$ is an even function then $\mu_{f}$ is symmetric.

Example 1. $P=U_{-a, a}(a>0)$ : Uniform distribution on $[-a, a]$. 
By simple computations, we have $\omega(t)=a(2 t-1), h(t)=a t(t-1)$ and $\gamma(t)=$ $a^{-1} \log \left(t^{-1}(1-t)\right) . \quad$ Therefore $\quad \gamma^{-1}(x)=\{1+\exp (a x)\}^{-1}$ and $f(x)=a \exp (a x)$ $\{1+\exp (a x)\}^{-2}$.

Example 2. $P=N^{\prime}(0,1)$ : Normal distribution with mean 0 and variance 1 .

Put $G(x)=(2 \pi)^{-1 / 2} \int_{-\infty}^{x} \exp \left(-x^{2} / 2\right) d x$. Then it is easy to see that $\omega(t)=G^{-1}(t)$, $h(t)=-(2 \pi)^{-1 / 2} \exp \left(-G^{-1}(t)^{2} / 2\right)$ and $\gamma(t)=-G^{-1}(t)$. Thus we have $\gamma^{-1}(x)=G(-x)$ and $f(x)=(2 \pi)^{-1 / 2} \exp \left(-x^{2} / 2\right)$.

Remark 3. As we have seen in Remark 1, a function $f$ which satisfies $\mu_{f}=P$ for a given $P$ is not unique. By the way we can take $f$ as an even function, if $P$ is symmetric. However such an even function is not uniquely determined as it will be seen in the following example.

Example 3. Put $g(x)=1 / 2|x| \exp (-|x|)$. Then $d \mu(x)=g(x) d x$ is a differentiable measure and after some calculations we have,

$$
\begin{aligned}
\mu\left(x \mid g^{\prime}(x) / g(x) \in E\right)= & 1 / 2 \int_{E}\left\{\frac{1}{(1+|x|)^{3}} \exp \left(-\frac{1}{1+|x|}\right)\right. \\
& \left.+\chi_{[-1,1]}(x) \frac{1}{(1-|x|)^{3}} \exp \left(-\frac{1}{1-|x|}\right)\right\} d x .
\end{aligned}
$$

Thus for the measure $P$ defined by the right hand side in the above equality, $g$ and $f$ obtained in the proof of Theorem are even solutions of $\mu_{f}=P$. However they does not coincide with each other, because $g(0)=0$ and $f(0)>0$.

\section{References}

[1] Hora, A., On a Banach space of functions associated with a homogeneous additive process, Publ. RIMS, Kyoto Univ., 24 (1988), 739-757.

[2] Yamasaki, Y., and Hora, A., Differentiable shifts for measures on infinite dimensional spaces, Publ. RIMS, Kyoto Univ., 23 (1987), 275-296.

[3] Shimomura, H., An aspect of differentiable measures on $R^{\infty}$, ibid., 23 (1987), 791811. 